\title{
Comparison of caffeine-induced changes in cerebral blood flow and middle cerebral artery blood velocity shows that caffeine reduces middle cerebral artery diameter
}

\author{
M J Lunt ${ }^{1}$, S Ragab ${ }^{2}$, A A Birch ${ }^{3}$, D Schley ${ }^{3}$ and D F Jenkinson ${ }^{2}$ \\ ${ }^{1}$ Department of Medical Physics, Royal Bournemouth Hospital, Castle Lane East, \\ Bournemouth BH7 7DW, UK \\ 2 Department of Medicine, Royal Bournemouth Hospital, Castle Lane East, \\ Bournemouth BH7 7DW, UK \\ ${ }^{3}$ Department of Medical Physics and Bioengineering, Southampton General Hospital, \\ Tremona Road, Southampton SO16 6YD, UK \\ E-mail: michael.lunt@rbch-tr.swest.nhs.uk
}

Received 16 September 2003, accepted for publication 23 January 2004 Published 17 February 2004

Online at stacks.iop.org/PM/25/467 (DOI: 10.1088/0967-3334/25/2/006)

\begin{abstract}
Changes in cerebral blood flow (CBF) can be assessed directly with xenon clearance $(\mathrm{XeC})$ or indirectly by measuring changes in middle cerebral artery blood velocity (Vmca) with transcranial Doppler (TCD). The aim of this study was to compare the changes in $\mathrm{CBF}$ and Vmca following caffeine ingestion.

Nineteen patients (age 48-86, recovering from an acute stroke) and ten controls (age 52-85) were each studied twice. Bilateral measurements of $\mathrm{CBF}$ and Vmca were made before and after ingestion of $250 \mathrm{mg}$ caffeine or matched placebo. The percentage change in CBF and Vmca after caffeine was calculated. Full results (CBF and Vmca) were obtained from 14 patients and 9 controls. There was no significant difference between patients and controls, so results were combined.

Caffeine reduced CBF by $22 \%$ (95\% confidence interval $(\mathrm{CI})=17 \%$ to $28 \%)$ and reduced Vmca by $13 \%(95 \% \mathrm{CI}=10 \%$ to $17 \%)$. The fall in Vmca was significantly less than that in $\mathrm{CBF}(p=0.0016)$, showing that caffeine reduces mca diameter. Analysis based on Poiseuille flow in the arterioles suggests that caffeine reduced arteriole diameter by $5.9 \%(95 \% \mathrm{CI}=4.6 \%$ to $7.3 \%)$ and mca diameter by $4.3 \%(95 \% \mathrm{CI}=2.0 \%$ to $6.6 \%)$.

$\mathrm{TCD}$ is being used as an alternative to $\mathrm{XeC}$ for assessing the effect of vasoconstrictors and vasodilators on CBF. This study has demonstrated that mca diameter can be changed by the vasoactive agents, and that changes in Vmca do not necessarily reflect changes in CBF.
\end{abstract}


Keywords: cerebral blood flow, blood flow velocity, caffeine, transcranial Doppler, xenon clearance, arterial diameter

\section{Introduction}

Changes in cerebral blood flow (CBF) can be assessed either directly with xenon clearance (XeC) or indirectly with transcranial Doppler (TCD) (Obrist and Wilkinson 1990, Newell and Aaslid 1992). XeC uses an array of gamma-ray detectors around the head to measure the rate of washout of radioactive xenon administered in the inhaled air. TCD uses an ultrasound probe on the side of the head to measure the time average of the instantaneous maximum blood velocity in the middle cerebral artery (Vmca). Fractional change in Vmca should be equal to fractional change in CBF provided vessel diameter and velocity profile remain constant (Kontos 1989).

It is well known that caffeine ingestion causes an acute reduction in CBF. Oral administration of $250 \mathrm{mg}$ caffeine to healthy young volunteers after at least $2 \mathrm{~h}$ abstinence reduced CBF by 18\% (Mathew and Wilson 1985) and intravenous infusion of $250 \mathrm{mg}$ of caffeine after $12 \mathrm{~h}$ abstinence reduced whole brain CBF by an average of 31\% (Cameron et al 1990). There is no evidence that this reduction in CBF causes any problems in the normal population in whom there is an adequate cerebral perfusion reserve, but there may be implications in ischaemic stroke patients. An ischaemic stroke is caused by a reduction in blood supply to an area of the brain leading to localized infarction, and there may be regions of hypo-perfusion around the infarct which could be vulnerable to any further flow reduction (Kaufmann et al 1999).

We have already used TCD to show that $250 \mathrm{mg}$ caffeine reduces Vmca by $13 \%$ in patients recovering from an ischaemic stroke in the middle cerebral artery (mca) territory (Lunt et al 2002). Caffeine of $250 \mathrm{mg}$ is equivalent to two $150 \mathrm{ml}$ cups of filter coffee or four cups of instant coffee or five to nine cups of tea depending on the brewing time (Bunker and McWilliams 1979). The aim of this study was to compare the changes in Vmca and CBF in stroke patients following caffeine ingestion to determine whether the change in Vmca does reflect a change in $\mathrm{CBF}$.

\section{Materials and methods}

Nineteen patients (age 48-86) and ten controls (age 52-85) were each studied twice using a randomized double-blind cross-over design. Patients were selected from the Stroke Rehabilitation Unit at Christchurch Hospital and from Acute Stroke Units at Royal Bournemouth and Southampton General Hospitals. All were recovering from an ischaemic stroke in the territory of a middle cerebral artery, diagnosis having been confirmed by clinical findings and consistent computed tomography scan appearances. The studies were performed at Southampton General Hospital. Ethical approval was obtained from the local Ethics Committees.

Before each study, subjects abstained from caffeinated drinks for $48 \mathrm{~h}$. Each study was performed with the subject lying supine in a quiet room. Two baseline CBF measurements and three baseline Vmca measurements were obtained bilaterally. Subjects were then given a capsule containing either $250 \mathrm{mg}$ of caffeine or placebo, and the CBF measurements repeated 40 and 60 min after administration. Four further Vmca measurements were obtained, two at $30 \mathrm{~min}$ and two at $90 \mathrm{~min}$ after administration. 
The CBF measurements were made using a Novo Cerebrograph 32c instrument, (manufactured by Novo Diagnostic Systems, Denmark). The subject inhaled air containing ${ }^{133} \mathrm{Xe}$ in a concentration of $110 \mathrm{MBq} \mathrm{l}^{-1}$ by means of a re-breathing administration system for $1 \mathrm{~min}$ and then continued to breathe normal air through the mask for the next 4 min to collect exhaust xenon. The rate of removal of the isotope from the brain was monitored throughout the $4 \mathrm{~min}$ and for a further 6 min by tracing the progressive change in radioactivity with a system of 16 collimated scintillation detectors mounted on a helmet. The impulse response function was determined by deconvolution with the curve of activity in the airway. CBF was calculated from the rate of washout of the impulse response using the initial slope index (Risberg and Prohovnik 1981).

The Vmca measurements were made using a transcranial Doppler unit (PCDop842, Scimed Ltd, Bristol, UK). The unit displayed the Doppler spectrum continuously, identified the maximum frequency envelope and converted frequency to velocity assuming blood flow was directly towards the probe. Once an optimum signal had been obtained, an $8 \mathrm{~s}$ sample was saved and the average of the maximum velocity envelope (Vmca) read from the TCD display. For each reading the operator manipulated the probe to find the maximum velocity signal through the temporal window. Each measurement consisted of three readings on each side.

At the end of each study, the subject was allowed to take their normal beverages until $48 \mathrm{~h}$ before any subsequent study. There was a gap of at least one week between studies to allow washout of any administered caffeine.

For each subject the mean CBF on each side of the brain before and after medication was calculated by averaging the results from the eight detectors on that side. The two initial measurements were then averaged to obtain the baseline CBF and the subsequent two measurements averaged to obtain the post-medication CBF. For the Vmca measurements, the maximum of each set of three readings was used. Baseline Vmca on each side was obtained by averaging the three measurements before medication, and post-medication Vmca obtained by averaging the four post-medication measurements.

After the code was broken, the effect of caffeine was found for each hemisphere separately by subtracting the change after placebo from the change after caffeine and these were averaged over all subjects. Overall percentage changes were calculated using averages of the premedication measurements as baselines. Statistical significance was assessed using Student's $t$-test with $5 \%$ significance level.

\section{Results}

Full CBF results (both sides and both visits) were available from 19 patients (mean age 71 years, range $48-86$ years) and 10 control subjects (mean age 69 years, range $52-85$ years). In some of these patients, it was not possible to obtain good TCD signals from both sides. Full Vmca results were available from only 14 patients (mean age 70, range 48-86) and 9 control subjects (mean age 68, range 52-85) (table 1 and figures 1 and 2).

In the patients, there was no significant difference between the affected and unaffected sides, so results from both were combined. Overall, the caffeine reduced CBF by $23.7 \%$ (SD 13\%, 95\% confidence interval 18\% to 30\%) and Vmca by $15.5 \%$ (SD 9\%, 95\% CI 10\% to 20\%). There was no significant correlation between the reduction in CBF and the age of the patient $(r=0.23, p>0.1)$.

In control subjects, there was no significant difference between the right and left sides so results from both were combined. Overall, the caffeine reduced CBF by $18.9 \%$ (SD 10\%, $95 \% \mathrm{CI}=12 \%$ to $26 \%$ ) and $\mathrm{Vmca}$ by $11.2 \%$ (SD $11 \%, 95 \% \mathrm{CI}=3 \%$ to $19 \%$ ). There 


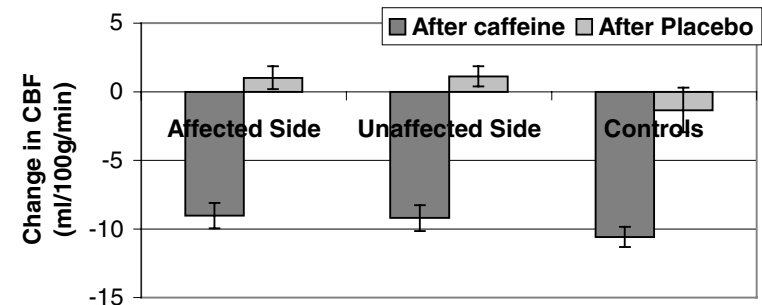

Figure 1. Change in CBF after tablet-patients and controls (mean \pm 1 SEM).

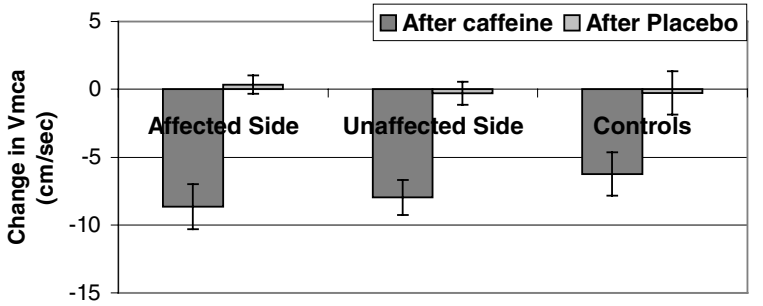

Figure 2. Change in Vmca after tablet-patients and controls (mean \pm 1 SEM).

Table 1. Summary of results.

\begin{tabular}{|c|c|c|c|c|}
\hline & \multicolumn{2}{|c|}{$\mathrm{CBF}\left(\mathrm{ml} / 100 \mathrm{~g} \mathrm{~min}^{-1}\right)$} & \multicolumn{2}{|c|}{$\operatorname{Vmca}\left(\mathrm{cm} \mathrm{s}^{-1}\right)$} \\
\hline & Affected side & Unaffected side & Affected side & Unaffected side \\
\hline \multicolumn{5}{|l|}{ (a) Patients } \\
\hline Baseline & 42.6 & 43.2 & 55.7 & 52.2 \\
\hline Fall after caffeine & 9.0 & 9.2 & 8.6 & 8.0 \\
\hline Fall after placebo & -1.0 & -1.1 & -0.4 & 0.3 \\
\hline Fall (caffeine-placebo) & 10.0 & 10.3 & 9.0 & 7.7 \\
\hline $95 \%$ confidence interval & $7.2-12.9$ & $7.9-12.8$ & $5.3-12.7$ & $5.4-9.9$ \\
\hline (b) Controls & Right side & Left side & Right side & Left side \\
\hline Baseline & 49.3 & 48.5 & 52.6 & 54.2 \\
\hline Fall after caffeine & 10.6 & 10.5 & 7.1 & 5.4 \\
\hline Fall after placebo & 1.3 & 1.4 & -1.6 & 2.1 \\
\hline Fall (caffeine-placebo) & 9.4 & 9.1 & 8.7 & 3.3 \\
\hline $95 \%$ confidence interval & $5.7-13.0$ & $5.4-12.9$ & $4.7-12.6$ & $2.7-9.2$ \\
\hline
\end{tabular}

was again no significant correlation between the reduction in CBF and the age of the subject $(r=0.10, p>0.5)$.

These results confirm that the reduction in Vmca does reflect a real reduction in CBF. However, it appears that the fall in Vmca is less than that in CBF. This was investigated in more detail by comparing the percentage reductions in Vmca and CBF in individual subjects. Full results were available from 23 subjects, 14 patients and 9 controls.

There was no significant difference between patients and controls, and between the affected and non-affected sides of the patients. Results were therefore combined. Caffeine reduced $\mathrm{CBF}$ by $22 \%$ (95\% confidence interval $(\mathrm{CI})=17 \%$ to $28 \%$ ) and reduced Vmca by $13 \%(95 \% \mathrm{CI}=10 \%$ to $17 \%)$. Comparison of the changes in individual subjects (figure 3 ) showed that the fall in Vmca was significantly less than that in $\operatorname{CBF}(p=0.0016)$. 


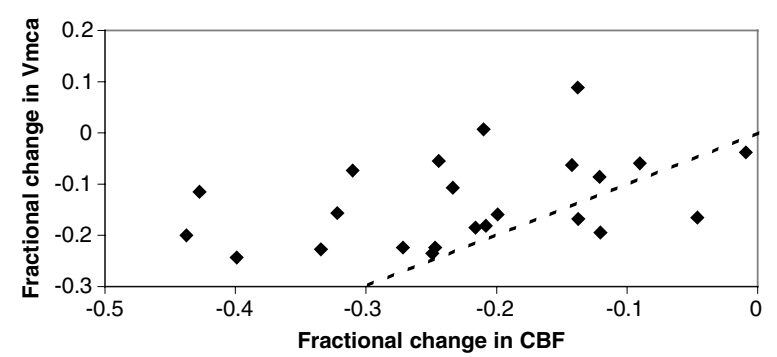

Figure 3. Comparison of fractional changes in Vmca and CBF in the individual subjects. The points do not lie on the line of identity (dotted line) and the fall in Vmca was significantly less than that in $\operatorname{CBF}(p=0.0016)$.

The consistency of the CBF and Vmca measurements was assessed by comparing measurements made on the same subject on the same occasion. The standard deviation of the difference between CBF measurements was $4.1 \mathrm{ml} / 100 \mathrm{gm} \mathrm{min}^{-1}$, and between Vmca measurements was $4.1 \mathrm{~cm} \mathrm{~s}^{-1}$. Baseline $\mathrm{CBF}$ and Vmca and their inter-subject standard deviations were estimated by comparing the pre-medication values in the different subjects. For CBF, baseline and standard deviation were 45 and $7.3 \mathrm{ml} / 100 \mathrm{gm} \mathrm{min}^{-1}$, and for Vmca they were 52 and $17.0 \mathrm{~cm} \mathrm{~s}^{-1}$.

\section{Discussion}

The different percentage changes in CBF and Vmca may arise because the two techniques assess different parts of the brain, which respond differently to caffeine. The middle cerebral artery supplies the parieto-temporal region of the brain, while the scintillation detectors were also positioned over the occipital region. CBF results from the 19 patients and 10 controls were therefore re-analysed using only the six parieto-temporal detectors on each side, but this made no significant change in the results. There was a $23 \%$ reduction in CBF compared to $22 \%$ when all detectors were used.

The CBF measurements will include more of the flow in the superficial cortex, while the middle cerebral artery supplies some deeper structures. Differences may occur if caffeine has a different effect on the grey and white matter and if there are different proportions of grey and white matter in these regions. The CBF measurements were re-analysed using a dual slope model (Obrist and Wilkinson 1990) to determine the effect on grey matter alone, but this made no significant change in the results. There was a $24 \%$ reduction in CBF to grey matter compared to $22 \%$ to grey and white combined. This is consistent with the findings of Field et al (2003) who used magnetic resonance imaging to study the effect of caffeine on $\mathrm{CBF}$ to grey and white matter, and found that $250 \mathrm{mg}$ caffeine reduced flow to grey matter by $23 \%$ and flow to white matter by $18 \%$.

Differences between changes in CBF and Vmca can also be caused by changes in velocity profile (Lunt et al 2000). However, profile changes will cause a larger change in Vmca than in CBF (Lunt et al 2000), so cannot account for the lower change in Vmca found in the present study.

Blood flow depends on the ratio of blood pressure and peripheral resistance. The reduction in CBF after caffeine must therefore be caused by a reduction in blood pressure, an increase in peripheral resistance, or a combination of the two. After caffeine, systolic blood pressure fell by $1.4 \%(2.0 \mathrm{mmHg}$ from a mean of $139 \mathrm{mmHg})$ and diastolic pressure fell by $0.7 \%$ 
( $0.5 \mathrm{mmHg}$ from a mean of $78 \mathrm{mmHg}$ ). These changes are far too small to account for the $22 \%$ fall in blood flow, so there must have been an increase in peripheral resistance.

The flow of an ideal fluid through a rigid tube is described by Poiseuille's law. Under the ideal conditions of steady established laminar flow, the resistance is proportional to the fourth power of the tube radius. Under these conditions, a $22 \%$ fall in flow would be caused by a $6 \%$ change in radius. Cerebral blood flow is controlled by arterioles, and many of the assumptions of the theoretical analysis will not strictly apply. In particular, the arterioles are elastic and the flow is pulsatile. In spite of these differences, our results (22\% change in CBF and $13 \%$ change in Vmca) are similar to those predicted by a steady-state model in which arteriolar and mca radii change by the same proportion. If arteriolar radius reduces by $6 \%$, the flow through the arterioles would fall by $22 \%$ and the flow through the mca would also fall by $22 \%$. But, if the mca radius also reduces by $6 \%$, the Vmca would only fall by $12 \%$, since the relation between velocity and flow would have changed. The validity of this model is discussed further in section 5 .

Even though the quantitative analysis may not be applicable, the difference between the reduction in flow and the reduction in velocity indicates that caffeine reduces middle cerebral artery diameter. This is an important result, since the use of transcranial Doppler as an alternative to xenon clearance is often justified by the assumption that there is no change in mca diameter.

There have been other studies examining whether vasoconstrictors or dilators change mca diameter. Huber and Handa (1967) used angiography to show that the diameters of cerebral arteries between 0.5 and $1.0 \mathrm{~mm}$ diameter were increased about $25 \%$ by the contrast agent (Urografin 60\%). Increasing $\mathrm{PaCO}_{2}$ from 40 to $57 \mathrm{mmHg}$ through $\mathrm{CO}_{2}$ inhalation or injecting $30 \mathrm{mg}$ papavarine also caused a $25 \%$ increase. However, the mca and vessels of similar calibre ( 2.0 to $2.5 \mathrm{~mm}$ diameter) were increased by a much smaller amount (4\% with $\mathrm{CO}_{2}$ inhalation). Giller et al (1993) obtained similar results by measuring vessel diameters directly using an operating microscope during craniotomy. End tidal $\mathrm{CO}_{2}$ was increased by an average of $14 \mathrm{mmHg}$, and this caused 18\% diameter increase in mca branches but only $2 \%$ in the mca itself.

There have also been other studies examining the relationship between changes in blood velocity and changes in flow. Dahl et al (1992) performed simultaneous TCD and XeC measurements to validate the use of TCD for cerebral vasoreactivity studies with acetazolamide as the stimulus. Intravenous administration of $1 \mathrm{~g}$ acetazolamide to 20 control subjects increased average CBF and Vmca by 30\% and 34\%, respectively. Demolis et al (1996) performed a similar study on 52 patients referred for cerebral vasoreactivity studies and found a 39\% increase in CBF and a $41 \%$ increase in Vmca. These results suggest that acetazolamide causes similar changes in CBF and Vmca. However, Brauer et al (1998) also used $1 \mathrm{~g}$ acetazolamide and found a 25\% increase in CBF and a $41 \%$ increase in Vmca. Although these results are somewhat inconsistent, there is no evidence that acetazolamide dilates both the arterioles and the mca, since this would cause a lower increase in Vmca than in CBF. This is important clinically, since acetazolamide is administered to stroke patients to assess cerebral vasoreactivity. The effect of the acetazolamide is assessed by measuring changes in $\mathrm{CBF}$ using $\mathrm{XeC}$ or changes in Vmca using TCD and the two have been shown to be equivalent (Dahl et al 1992).

In summary, different vasodilators and vasoconstrictors have different effects on the mca diameter. Acetazolamide appears to have little effect, carbon dioxide has some effect and caffeine may have a greater effect, with the percentage change in mca radius comparable to that in the flow-regulating vessels. However, if caffeine does have a consistent effect on all vessels, there should be a consistent relationship between the changes in flow and velocity in individual subjects. This is investigated below. 


\section{Comparison of changes in velocity, flow and pressure in individual subjects}

We have suggested above that caffeine may be causing the same percentage reduction in the radius of mca and arterioles. This was based on the application of Poiseuille's formula,

$$
Q=\frac{\pi}{8 \eta} \frac{\partial P}{\partial L} r^{4}
$$

where $Q$ is the flow through a straight, rigid capillary tube; $\eta$ the viscosity of the fluid, $\partial P / \partial L$ the pressure gradient and $r$ is the radius of the tube.

This formula will not strictly apply to arterioles because they are branched, curved, elastic and carry pulsatile flow, but it suggests that mean flow in arterioles may be proportional to (pressure gradient) $\times r^{4}$. There is only a small pressure drop in vessels proximal to the arterioles, so the pressure gradient can be assumed to be proportional to the difference between arterial blood pressure (ABP) and venous pressure. If cerebral venous pressure is assumed to be zero, pressure gradient will be proportional to $\mathrm{ABP}$ and mean flow in the arterioles will be proportional to $\mathrm{ABP} \times r^{4}$. Hence, if $q, P$ and $r$ are the $\mathrm{CBF}, \mathrm{ABP}$ and arteriole radius in a subject and suffices 1 and 2 are before and after medication (caffeine or placebo),

$$
\left(\frac{r_{2}}{r_{1}}\right)^{4}=\frac{q_{2}}{q_{1}} \frac{P_{1}}{P_{2}} \text {. }
$$

This can be used to find the medication-related change in arteriole radius $r$ in a given subject. It is important to include the blood pressure, since changes in individual subjects were as high as $15 \mathrm{mmHg}$ between the pre-caffeine and post-caffeine measurements, although the average change across all subjects was less than $1.5 \mathrm{mmHg}$.

Mca blood flow $Q$, radius $R$ and mean blood velocity $V_{\text {mean }}$ are related by

$$
Q=\pi R^{2} V_{\text {mean }}
$$

Transcranial Doppler measures the time-averaged maximum velocity $(V)$ instead of the mean. The exact relationship between these depends on the velocity profile (Lunt et al 2000) but they will be proportional if the profile remains constant. In this case,

$$
\left(\frac{R_{2}}{R_{1}}\right)^{2}=\frac{Q_{2}}{Q_{1}} \frac{V_{1}}{V_{2}}
$$

where subscripts 1 and 2 denote before and after medication.

Assuming that the percentage changes in flow in the arterioles and mca are equal to the percentage change in CBF, equations (1) and (2) can be used to examine the changes in arteriole and mca radii $\left(r_{2} / r_{1}\right.$ and $R_{2} / R_{1}$ respectively) after caffeine and placebo. Results are given in table 2. Caffeine causes significant changes in arteriole radius $\left(r_{2} / r_{1}=0.941\right.$, $p<0.001)$ and in mca radius $\left(R_{2} / R_{1}=0.957, p<0.001\right)$. Changes after placebo are not statistically significant.

Comparison of $r_{2} / r_{1}$ and $R_{2} / R_{1}$ in individual subjects does not provide hard evidence that these ratios are the same. In fact, the hypothesis that they are the same can be rejected at the $10 \%$ significance level $(p=0.08)$.

Table 2. Mean fractional change in vessel radius assuming Poiseuille flow in the arterioles. Both caffeine-induced changes are statistically significant.

\begin{tabular}{lllll}
\hline & $\begin{array}{l}\text { Mca } \\
\text { (mean change) }\end{array}$ & $\begin{array}{l}\text { Mca } \\
(95 \% \mathrm{CI})\end{array}$ & $\begin{array}{l}\text { Arteriole } \\
(\text { mean change })\end{array}$ & $\begin{array}{l}\text { Arteriole } \\
(95 \% \mathrm{CI})\end{array}$ \\
\hline After caffeine & 0.957 & $0.934-0.980$ & 0.941 & $0.927-0.954$ \\
After placebo & 1.004 & $0.982-1.026$ & 0.996 & $0.979-1.013$ \\
\hline
\end{tabular}


The above analysis, based on Poiseuille flow in the arterioles, therefore suggests the $250 \mathrm{mg}$ caffeine reduces arteriole diameter by $5.9 \%$, and mca diameter by $4.3 \%$. Both changes are statistically significant.

\section{Conclusions}

This study has demonstrated that $250 \mathrm{mg}$ caffeine reduces CBF by an average of $22 \%$ in healthy volunteers and in patients recovering from an acute ischaemic stroke, and that both cerebral hemispheres are affected equally. The caffeine causes a significantly smaller change of $13 \%$ in Vmca, and this shows that caffeine reduces the mca diameter. Analysis based on Poiseuille flow in the arterioles suggests that the $250 \mathrm{mg}$ caffeine reduced arteriole diameter by $5.9 \%$, and mca diameter by $4.3 \%$.

Transcranial Doppler is being used as an alternative to xenon clearance for assessing the effect of vasoconstrictors and vasodilators on cerebral blood flow. This study has demonstrated that mca diameter can be changed by the vasoactive agents, and that changes in Vmca do not necessarily reflect changes in $\mathrm{CBF}$.

\section{References}

Brauer P, Kochs E, Werner C, Bloom M, Policare R, Pentheny S, Yonas H, Kofke W A and Schulte am Esch J 1998 Correlation of transcranial Doppler sonography mean flow velocity with cerebral blood flow in patients with intracranial pathology J. Neurosurg. Anesthesiol. 10 80-5

Bunker M L and McWilliams M 1979 Caffeine content of common beverages J. Am. Diet. Assoc. 74 28-32

Cameron O G, Modell J G and Hariharan M 1990 Caffeine and human cerebral blood flow: a positron emission tomography study Life Sci. 47 1141-6

Dahl A, Lindegaard K-F, Russell D, Nyberg-Hansen R, Rootwelt K, Sorteberg W and Nornes H 1992 A comparison of transcranial Doppler and cerebral blood flow studies to assess cerebral vasoreactivity Stroke 23 15-9

Demolis P, Tran Dinh Y R and Giudicelli J-F 1996 Relationships between cerebral regional blood flow velocities and volumetric blood flows and their respective reactivities to acetazolamide Stroke 27 1835-9

Field A S, Laurienti P J, Yen Y-F, Burdette J H and Moody D M 2003 Dietary caffeine consumption and withdrawal: confounding variables in quantitative cerebral perfusion studies? Radiology 227 129-35

Giller C A, Bowman G, Dyer H, Mootz L and Krippner W 1993 Cerebral arterial diameters during changes in blood pressure and carbon dioxide during craniotomy Neurosurgery 32 737-42

Huber P and Handa J 1967 Effect of contrast material, hypercapnia, hyperventilation, hypertonic glucose and papavarine on the diameter of the cerebral arteries. Angiographic determination in man Invest. Radiol. 2 17-32

Kaufmann A M, Firlik A D, Fukui M B, Wechsler L R, Jungries C A and Yonas H 1999 Ischemic core and penumbra in human stroke Stroke 30 93-9

Kontos H A 1989 Validity of cerebral artery blood flow calculations from velocity measurements (editorial) Stroke 20 1-3

Lunt M J, Jenkinson D F and Kerr D 2000 Transcranial Doppler blood velocity measurement — the effect of changes in velocity profile Ultrasound Med. Biol. 26 1145-51

Lunt M J, Hanrahan A, Kerr D and Jenkinson D F 2002 Measuring caffeine-induced changes in middle cerebral artery blood velocity using transcranial Doppler in patients recovering from ischaemic stroke Physiol. Meas. 23 375-83

Mathew R J and Wilson W H 1985 Caffeine induced changes in cerebral circulation Stroke 16 814-7

Newell D W and Aaslid R 1992 Transcranial Doppler: clinical and experimental uses Cerebrovasc. Brain Metab. Rev. 4 122-43

Obrist W D and Wilkinson W E 1990 Regional Cerebral Blood Flow Measurement in Humans by Xenon-133 Clearance Cerebrovasc. Brain Metab. Rev. 2 283-327

Risberg J and Prohovnik I 1981 rCBF measurements by 133-Xenon inhalation: recent methodological advances Prog. Nucl.Med. 7 70-81 